\title{
Regarding 'Modifications of chest radiography exposure parameters using a neonatal chest phantom' by Schäfer et al.
}

\author{
Karl O. Schneider ${ }^{1}$ (1)
}

Received: 18 May 2020 / Revised: 18 May 2020 / Accepted: 6 July 2020 / Published online: 24 July 2020

(C) Springer-Verlag GmbH Germany, part of Springer Nature 2020

\section{Dear Editors,}

The paper "Modifications of chest radiography exposure parameters using a neonatal chest phantom" by Schäfer and coworkers [1] deals with an important issue in neonatal radiography. However, it is questionable whether optimisation of the radiographic technique can be reached with the Gammex 610 Neonatal Chest Phantom. In my opinion, this phantom is not suitable to evaluate critical anatomical lung structures in neonates and infants because it is a fixed phantom. In a real chest radiograph, there are many moving structures (e.g., heart, diaphragm, pulmonary interstitial structures), all of which can cause blurring artefacts. I wonder why the exposure time was not registered in the study design. Short exposure time is essential to avoid moving artefacts. The European Guidelines EUR 16261 recommend an exposure time of less than $4 \mathrm{~ms}$. If the kilovoltage is lowered below $60 \mathrm{kVp}$, the current-time product (mAs) must be increased even with full digital systems. On the other hand, the increased $\mathrm{mAs}$ would lead to longer exposure time and consequently more motion artefacts. Another type of artefacts is caused by the pulsation of the great vessels (aorta, pulmonary artery), which can substantially degrade the visibility of the distal trachea and the left mainstem bronchus. This image criterion is very important for not missing severe diseases concerning the trachea and great vessels. If we look closely at the image series, the trachea and the mainstem stem bronchus can be identified only with 63
$\mathrm{kVp}$, but with only $1.62 \mathrm{mAs}$, using $40 \mathrm{kVp}$, the large airways are not visible. In addition, the brightness and the noise in the lungs are very high and simulate interstitial lung pathology; the opposite is true for the high $\mathrm{kVp}$ series. The inconclusive results of this study reassure us of the recommendations of the European Guidelines EUR 16261. Last, a short remark on the collimation of the images: All chest images are centred too low, i.e. the lateral parts of the clavicles are not displayed. In my view, the Gammex phantom may be useful if different detector systems and post-processing tools are compared, but not if the radiographic technique of infant chest radiographs has to be optimised.

\section{Compliance with ethical standards}

Conflicts of interest None

\section{References}

1. Schäfer SB, Papst S, Fiebich M et al (2020) Modification of chest radiography exposure parameters using a neonatal chest phantom. Pediatr Radiol 50:28-37

Publisher's note Springer Nature remains neutral with regard to jurisdictional claims in published maps and institutional affiliations.
Karl O. Schneider

Karl.Schneider@med.uni-muenchen.de

1 Department of Pediatric Radiology,

Dr. von Hauner's Children Hospital, University of Munich LMU, Lindwurmstr. 4, 80337 Munich, Germany 